\title{
Medición de la innovación agropecuaria desde los territorios: una propuesta conceptual y metodológica
}

\section{Measurement of agricultural innovation from the territories: a conceptual and methodological approach}

\author{
Jairo Rojas-Meza \\ Universidad Nacional Autónoma de Nicaragua, UNAN-Managua, FAREN- Matagalpa,jrojas_12@yahoo.com
}

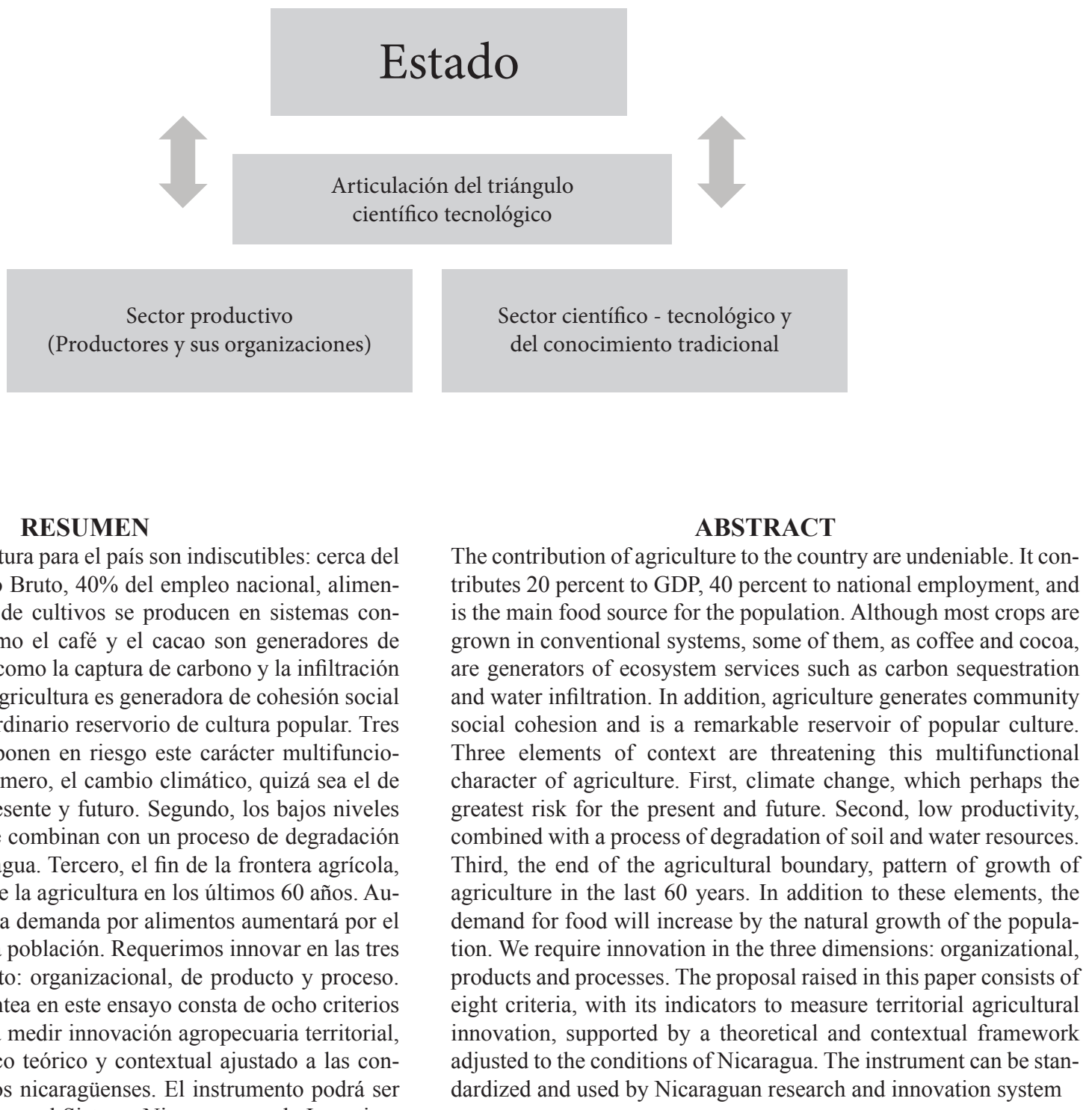

Los aportes de la agricultura para el país son indiscutibles: cerca del $20 \%$ al Producto Interno Bruto, 40\% del empleo nacional, alimentos; aunque la mayoría de cultivos se producen en sistemas convencionales algunos como el café y el cacao son generadores de servicios ecosistemicos como la captura de carbono y la infiltración de agua. Así mismo, la agricultura es generadora de cohesión social comunitaria y un extraordinario reservorio de cultura popular. Tres elementos de contexto ponen en riesgo este carácter multifuncional de la agricultura. Primero, el cambio climático, quizá sea el de mayor riesgo para el presente y futuro. Segundo, los bajos niveles de productividad, que se combinan con un proceso de degradación de los recursos suelo y agua. Tercero, el fin de la frontera agrícola, patrón del crecimiento de la agricultura en los últimos 60 años. Aunado a estos elementos la demanda por alimentos aumentará por el crecimiento natural de la población. Requerimos innovar en las tres dimensiones del concepto: organizacional, de producto y proceso. La propuesta que se plantea en este ensayo consta de ocho criterios con sus indicadores para medir innovación agropecuaria territorial, sustentados por un marco teórico y contextual ajustado a las condiciones de los territorios nicaragüenses. El instrumento podrá ser normalizado y utilizado por el Sistema Nicaragüense de Investigación e Innovación Agropecuaria.

Palabras clave: innovación territorial agropecuaria, sistema de innovación. 


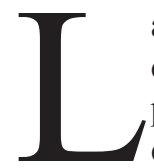

a innovación en todas las dimensiones de su definición, es una tarea crucial para avanzar en la competitividad y los propósitos del desarrollo humano en los territorios y países. Nicaragua no cuenta con un sistema de indicadores que mida la innovación en general y la agropecuaria en los territorios en particular, siendo una limitante para conocer los procesos, factores y resultados de políticas y planes. En el campo internacional, especialmente desde la Organización para la Cooperación y Desarrollo Económico (OCDE) se han desarrollado instrumentos para conceptualizar y operacionalizar la medición de la innovación. El Manual Frascati publicado por primera vez en 1963 y el Manual de Oslo en 1993, reflejan el interés de los gobiernos y la comunidad científica de contar con instrumentos homologados para medir el desempeño de la ciencia, la tecnología y la innovación. Recientemente se publicó el Manual del Bogotá, orientado a la normalización de indicadores de innovación tecnológica más contextualizados a las realidades de América Latina y el Caribe.

Otros autores como Cancino (2008) RESINDEX (2008) González (2010) han construido propuestas de indicadores para medir la innovación desde los territorios, teniendo como base teórica las contribuciones acerca de los sistemas regionales de innovación de Lundvall, 1988; Nelson, 1993; Patel y Pavitt, 1993.

Contar con un sistema de indicadores de la innovación es fundamental en los procesos de su gestión, por ser un valioso instrumento para la evaluación e incidencia en las políticas, planes, programas y acciones del sector público y el privado. Contar con micro, pequeñas y medianas empresas, productores y territorios innovadores supone no solo mayor competitividad, sino también la generación de dinámicas virtuosas hacia los restantes agentes económicos, con su consecuente influencia en el desarrollo del país.

Este ensayo defiende la idea que los esfuerzos mencionados, si bien representan un referente importante, no se adecúan al marco conceptual que explica la realidad de la agricultura en los territorios nicaragüenses, ni a los desafíos en materia de ciencia, tecnología e innovación para un modelo de agricultura propio, ecológicamente sustentable, viable desde la dimensión económica y socialmente justo. Así mismo, no toman en cuenta el reconocimiento cada vez más generalizado de la existencia de múltiples fuentes y actores generadores de conocimiento para la innovación, como el ancestral y comunitario, por tanto, la necesidad de emprender un proceso de dialogo de saberes.

El planteamiento anterior se circunscribe en la afirmación del Manual de Bogotá en el sentido que el seguimiento de los procesos innovativos debe apuntar no sólo a conocer las magnitudes, es decir, los aspectos cuantitativos, sino también las características de esos procesos, con el propósito de obtener evidencias respecto del sendero de desarrollo por el que transita una economía, aspecto que adquiere un enorme valor estratégico en la formulación de políticas públicas nacionales, sectoriales y locales.

La definición de un conjunto de indicadores sobre la innovación del sector agropecuario permitirá al naciente Sistema Nicaragüense de Investigación e Innovación Agropecuaria (SNIA) contar con un instrumento que podrá ayudar a los procesos para su gestión. Este instrumento podría facilitar la definición de la línea de base para conocer la situación de la innovación en los territorios del país, el monitoreo y evaluación de los planes, proyectos y acciones implementados por los protagonistas - productores, gobierno e instituciones de formación e investigación en materia de innovación agropecuaria.

A continuación se presenta el marco conceptual que fundamenta al sistema de indicadores propuesto, el cual fue construido considerando la realidad de la agricultura en los territorios nicaragüense, la apuesta por un nuevo modelo de innovación sustentado en la interacción de múltiples actores, saberes y factores, así como una agricultura sustentada en el conocimiento de la naturaleza, sus múltiples funciones, ambientales y sociales.

El marco conceptual de la innovación. El concepto de la innovación es considerada en este trabajo desde tres dimensiones: organizacional, como proceso y resultado. La innovación organizacional está referida al diseño e institucionalización de políticas, estrategias, y acuerdos entre sus principales actores. Se refiere también, al establecimiento de mecanismos organizacionales no coyunturales, desde la identificación participativa de las demandas de los productores, el análisis prospectivo de la agricultura y su incidencia en los territorios, la búsqueda dialogada de opciones dentro del bagaje existente: explícito - tácito, la investigación básica - aplicada, la gestión colectiva del conocimiento mediante redes de aprendizaje, y la adopción de prácticas y tecnologías.

Desde la perspectiva de procesos y resultado, la innovación se refiere a la incorporación de nuevos productos, la mejora de los ya existentes o de los procesos para su generación. Brunet y Baltar (2010) afirman que la innovación no solo incluye los grandes cambios, sino también los incrementales. Se considera como innovación incremental los pequeños cambios o mejoras en la tecnología existente, y como innovación radical, un cambio completo en la dirección del proceso innovador, pudiendo producirse una destrucción creadora que deje obsoletas las tecnologías anteriores. Quintero (2009) considera la innovación como proceso se refiere a la manera en que ha sido creado y elaborado un producto, a las etapas que conducen a su fabricación.

La innovación es un elemento central en las estrategias de desarrollo, definido como un proceso dinámico de interacción que une agentes como lo productores, sus organizaciones, las instituciones del Estado y de formación e investigación agropecuaria. Los vínculos sistemáticos y la interacción 
entre actores, así como la infraestructura económica e institucional que cada país es capaz de desarrollar, determinan la posibilidad de avanzar hacia una cultura del emprendimiento e innovación, fundamentales para impulsar círculos virtuosos de desarrollo (CEPAL, 2006). Los desarrollos teóricos se han preocupado por identificar si se trata de un proceso lineal o multidireccional, de ahí se derivó la importancia de observar la innovación como un proceso interactivo entre agentes internos y externos a las empresas o territorios (Quintero, 2009).

El concepto de innovación ha evolucionado desde una perspectiva centrada en la empresa hacia otra fundamentada en el territorio y los múltiples actores. La primera es la Shumpeteriana, sustenta la innovación como base de su teoría del desarrollo económico. Consideraba que las innovaciones surgían del interior de las grandes empresas debido a su actividad investigadora. Desde finales de la Segunda Guerra Mundial hasta mediados de la década de los años 70s del Siglo XX, esta concepción va a impregnar las políticas de desarrollo exógeno centradas en la gran empresa, estandarizada y basada en la oferta de los incentivos del Estado a las grandes empresas. Se suponía que el conocimiento codificado y las innovaciones se trasferían siguiendo una trayectoria lineal y unidireccional, desde centros de investigación y universidades estatales hacia las empresas (Brunet y Baltar, 2010).

El modelo lineal de innovación asumía que la tecnología es información fácil de copiar y en la medida en que las innovaciones se pueden codificar como información, se transforman en bienes y servicios cuya difusión es inmediata y barata, mediante la imitación y copia. Sin embargo, como expresan los autores, los mecanismos a través de los cuales surgen las innovaciones son más complejos.

La segunda perspectiva es la evolucionista, caracteriza a las innovaciones como iniciativas empresariales intencionales para romper con el equilibrio de los mercados perfectos y lograr el monopolio temporal.

Tanto el modelo Shumpeteriano como el evolucionista no son aplicables a las características en la agricultura, especialmente la pequeña y mediana, que es la predominante en Nicaragua. La cual en general no se rige estrictamente por la lógica del mercado, por ser unidades de producción y consumo. Por otro lado, las escalas de producción no les permiten contar con estructuras de investigación e innovación, como se plantea en los modelos mencionados.

Estudios recientes en diversos países de América Latina, han demostrado que en general en la agricultura, los procesos de innovación no responden al patrón lineal originado en el paradigma de la gran empresa, sino como resultado de múltiples interacciones. Por ejemplo, Muñoz et al., (2004) encontraron que la dinámica de innovación entre citricultores del Valle mexicano de Apatzingán no se explicaba mediante el enfoque lineal, según la cual, de la investigación básica se pasa a la aplicada, después al desarrollo tecnológico y posteriormente a su difusión. Más bien, dicha dinámica era el resultado de múltiples interacciones, entre numerosos y diversos actores: los citricultores, los proveedores de tecnologías, los asesores, los empacadores y los investigadores, entre otros.

El actor dominante en esta dinámica de interacción son los propios citricultores, considerando que el 70\% del conocimiento y la innovación han provenido de la comunicación entre ellos, lo que significa que el tipo de aprendizaje es el denominado "imitación por duplicación”. El cual si bien ha permitido difundir un conjunto de conocimientos útiles en un tiempo relativamente corto y un bajo costo, presenta una serie de debilidades dada su fuerte dosis de espontaneidad y empirismo. Basado en la difusión de conocimientos tácitos, esta forma de aprendizaje resulta útil para cubrir los requerimientos inmediatos del proceso productivo, pero en ausencia de procesos de aprendizaje de "imitación creativa" y de "verdadera innovación", se corre el riesgo de erigir una actividad económica movida exclusivamente por la inercia y sujeta a enormes riesgos ante cualquier cambio en el entorno (Muñoz et al., 2004).

A continuación se analiza el enfoque de la innovación desde la perspectiva de sistemas, el cual está siendo considerado como el paradigma para acelerar la adaptación de la agricultura a los desafíos contemporáneos como: el cambio climático, pobreza, globalización, seguridad alimentaria y la degradación de los recursos naturales, especialmente el suelo y agua.

Los sistemas de innovación en la agricultura (SIA). Los sistemas de innovación tienen sus antecedentes en el análisis crítico del pensamiento Latinoamericano que analiza la problemática de ciencia - tecnología e innovación. (Sabato, 1975) uno de los principales exponentes de la dependencia científica de nuestros países, planteó que entre las debilidades del proceso de desarrollo era la desarticulación de lo que llamó el triángulo científico - tecnológico. Souza (2005) opina que el modo clásico de innovar para el desarrollo está en crisis. Para este autor, el modo clásico eurocéntrico bajo el cual unos generan, otros transfieren y muchos adoptan, sin interacción entre ellos está en decadencia. Las premisas que sostienen su propuesta epistémico - ideológica son insatisfactorias para interpretar y transformar la realidad compleja, por su descontextualización.

El enfoque de los Sistemas de Innovación es una apuesta para superar las limitaciones de la innovación, en el cual las empresas e instituciones de investigación realizan la incorporación de tecnologías siguiendo el patrón lineal: investigación básica, aplicada, validación, transferencia, adopción e incorporación de los productos o servicios al mercado. Este modelo por su funcionamiento no reconoce las variadas fuentes de conocimiento y el papel de la interacción de múltiples actores en el proceso de innovación. El eje central es 
la complementariedad entre los subsistemas, para producir, difundir y utilizar el conocimiento, siendo el sistema más que la suma de las partes. Según Souza (2005) la ciencia moderna no es la única vía válida para la generación de conocimiento relevante; existen otras posibilidades paradigmáticas y otros saberes útiles para la transformación social y productiva.

Los procesos de innovación que impactan positivamente en el nivel de competitividad y en la tasa de crecimiento, no son generados por instituciones de I $+\mathrm{D}$ trabajando de forma aislada respecto al mercado, sino que son producidas para la solución en contexto de aplicación, y mediante la interacción de múltiples agentes e instituciones (Brunet y Bal$\operatorname{tar}, 2010)$.

El GRAS (2012) reconoce que el Sistema de Innovación vuelve la atención hacia la necesidad de entender las corrientes de tecnología e información entre las personas, empresas e instituciones y reconocer como se dan las innovaciones. La evaluación de estos sistemas significa mirar los efectos directos en términos de procesos de cambio tecnológico e institucional. También implica hacer preguntas críticas acerca de la pertinencia de la teoría social del cambio, mediante la construcción e implementación de los sistemas de innovación.

Cotec (2005) argumenta que de la interacción de los protagonistas de un sistema de innovación se esperan los siguientes resultados; De los productores con: a) la administración pública o el Estado; financiamiento, ayudas no financieras, regulación, mercado público de la tecnología, definición de políticas científicas y tecnológicas de innovación, incentivos que fomente la $\mathrm{I}+\mathrm{D}+\mathrm{i}$ en los sectores de menor productividad; b) el Sistema Público de $I+D+i$ : alineación de la estrategia de investigación pública con el tejido empresarial, transferencia de tecnología entre sistemas I + D + i y la empresa, entornos que propicien el intercambio de conocimientos, movilidad de personal, impulso a la creación de empresas de base tecnológica; c) las organizaciones de soporte a la innovación: desarrollo de tecnologías de base, alianzas estratégicas, I + D bajo contrato, servicios para la innovación a través de parques científicos y tecnológicos, movilidad de personal, entre otros.

Así mismo este autor, en las relaciones entre la administración y el sistema público científico de $\mathrm{I}+\mathrm{D}+\mathrm{i}$, identifica las posibilidades de formular estrategia científica y tecnológica, captación de investigadores en el mercado internacional, valorización social de la actividad investigadora, creación y mantenimiento de las infraestructuras, financiamiento de programas compartidos.

Lo relevante del planteamiento de Cotec (2005) es que identifica un conjunto de posibilidades para las alianzas entre los protagonistas de un sistema de innovación, que facilitaría la articulación del llamado triángulo científico - tecnológico, término acuñado por Sabato (1975). Desde esta perspectiva la estrategia de innovación está orientada a la conformación de redes, que configuran una alianza estratégica permanente hacia el desarrollo competitivo de sus integrantes. Estos mecanismos de integración permiten el fácil intercambio de información, adquirir y transmitir conocimientos, constituyendo un flujo de entrada para mejorar los procesos de aprendizaje e innovación.

Entre las principales experiencias internacionales en la implementación del modelo de gestión de la innovación en esta concepción, encontramos los Sistemas de Conocimiento e Innovación Agrícola (AKIS) de los países que conforman la Unión Europea, que tiene como base la coproducción de innovaciones mediante la interacciones entre todos los involucrados en las cadenas alimenticias, especialmente para lo que ellos han llamado cambio de segundo orden "innovación sistémica, como la introducción de la agricultura multifuncional. Los AKIS se definen como un conjunto de organizaciones o personas y los vínculos e interacciones entre ellos, dedicadas a la generación, transformación, transmisión, almacenamiento, difusión y utilización de los conocimientos para respaldar la toma de decisiones, la resolución de problemas y la innovación en la agricultura (Roling y Engel, 1991).

En América Latina, Perú ha logrado institucionalizar un Sistema de Innovación Agropecuario (SNIA), el cual fue aprobado por Decreto Legislativo orientado a promover el desarrollo de la investigación, el desarrollo tecnológico, la innovación y la transferencia de tecnología en materia agraria con la finalidad de impulsar la modernización agraria y la competitividad del sector. El SNIA está conformado por el conjunto de instituciones, principios, normas, técnicas e instrumentos, mediantes las cuales el Estado articula el esfuerzo innovador de las instituciones del sector público agropecuario, las universidades, instituciones de educación técnicas y las organizaciones de productores.

En Nicaragua, a inicios del año 2015 se conformó el Sistema Nicaragüense de Investigación e Innovación Agropecuaria como un mecanismo de concertación, coordinación, planificación, implementación, seguimiento y evaluación de la actividad de investigación e innovación agropecuaria del país, que tiene como propósito contribuir al desarrollo de la capacidad productiva. La base organizativa se sustenta en una alianza entre el sector público de investigación, representada por el INTA, las instituciones de formación e investigación pertenecientes al Consejo Nacional de Universidades, las organizaciones de productores, el Tecnológico Nacional y como invitadas permanentes las instituciones internacionales de investigación y cooperación científica y técnica con presencia en el país.

Existen otras experiencias de redes o plataformas de cooperación multi actores con resultados promisorios en términos de innovación agropecuaria, promovidos por proyectos regionales para Centroamérica como la RED SICTA y el PRIICA, que están dejando aprendizajes relevantes acerca del proceso de innovación mediante sistemas territoriales. De 
México, podemos citar las experiencias de las redes de innovación promovidas por la Fundación Produce, especialmente en el Estado de Michoacán, que muestran el aumento en la velocidad de la innovación cuando se actúa mediante estos mecanismos de interacción.

La construcción de territorios que aprenden e innovan. Diversos autores han tratado de asemejar al territorio como un organismo o entidad inteligente, que aprende e innova. Evidentemente no son los territorios como espacios físicos quienes tienen estas propiedades, sino las personas, las organizaciones y los arreglos institucionales que se construyen y gestionan. El territorio como una construcción social es un espacio propicio para emprender procesos de innovación. Brunet y Baltar (2010) afirman que en los procesos de desarrollo han de intervenir los agentes económicos, sociales e institucionales que forman el medio en el que se desarrolla la actividad productiva y crean un nexo de relaciones productivas, comerciales, tecnológicas y culturales, cuya densidad y carácter innovador favorece los procesos de crecimiento y desarrollo endógeno.

Para Cooke (2001) la innovación es un proceso social y técnico. Distingue dos tipos de aprendizaje: a) aquellos generados en el propio proceso de producción, mediante la experiencia y b) los basados en la investigación científica, el descubrimiento o la recombinación de paradigmas tecnológicos existentes. Con estos conceptos, el autor sugiere una metodología para identificar las regiones con desarrollo de conocimiento intensivo en ciencia y otro para las regiones que se encuentran más rezagadas. Bajo esta perspectiva, como expresa Viotti (2001) los países, regiones y territorios pueden tener sistemas de aprendizajes pasivos o activos.

La incorporación de activos de conocimiento reflejan, además, la capacidad dinámica de aplicar y materializar el conocimiento adquirido interna y externamente, en la obtención de nuevos productos, métodos de producción, distribución y de comercialización (Vega et al., 2009). La innovación como proceso territorial se fundamenta en las siguientes premisas: a) el aprendizaje como proceso localizado; b) la innovación como un proceso de aprendizaje interactivo, que implica poner énfasis en la cooperación; c) las redes, la coordinación, los acuerdos entre los actores para construir una agenda sustantiva que permita compartir y crear conocimiento relevante a las necesidades de los sistemas productivos.

Los Sistemas Regionales o territoriales de innovación han sido considerados mecanismos para asumir la heterogeneidad entre las regiones de un país y no suponer que los Sistemas Nacionales de Innovación interpretan totalmente esta realidad. Según Lundvall (1992) se trata de una alternativa metodológica para resolver ese vacío e interpretar la dinámica de la innovación en el ámbito geográfico de la región. Esto requiere en países como Nicaragua avanzar en la descentralización geográfica y política de los sistemas de formación básica, técnica, universitaria y la especialización sectorial de los sistemas productivos.
Factores asociados con la innovación en la agricultura. La innovación en el sector agropecuario y la identificación de los aspectos que la determinan son un desafío que en la actualidad enfrenta la sociedad. Los estudios de la innovación en la agricultura han transitado por diversos momentos e interpretaciones. Algunos estudios esclarecen interrogantes sobre que estimula a un productor para que desarrolle, adopte o realice alguna innovación o cuales son los elementos que provocan su rechazo.

La mayoría de los estudios sobre los factores asociados a la innovación se han realizado especialmente en el ámbito de las micro, pequeñas y medianas empresas asentadas en los espacios urbanos. Hacen una clasificación entre factores internos y externos. En el primero, consideran la innovación como resultado de actuaciones en la propia empresa y dependen de la propia capacidad de dirección para consolidar las dimensiones de calidad y productividad, así como la posibilidad de crear a menor costo, y más rápidamente que los competidores: tecnologías, competencias y actitudes esenciales que generen productos y servicios innovadores (Hidalgo et al., 2008). Por otro lado, Da Casta et al., (2014) identificaron que la complejidad, autonomía, integración organizacional, recursos organizacionales, cultura de organización horizontal, cooperación entre empresas, el liderazgo positivo de apoyo a la participación y creatividad estaban asociados a la innovación. Dentro de los factores externos para la innovación identifican el contexto institucional, políticas de apoyo al sector y las características de las políticas económicas.

Para Muñoz et al., (2009) el enfoque de redes o de sistemas de innovación sectoriales o territoriales permite mejorar el intercambio de información y conocimientos. En ese sentido mejorar el patrón de comunicación entre los miembros de una red, facilita la rapidez con la cual se pueden difundir y ser adoptadas las innovaciones. Conocer dicho fenómeno es fundamental para la comprensión de la innovación desde estos mecanismos sociales.

Abadi y Pannel (1999) afirman que a pesar de los numerosos estudios, los resultados de la investigación y los modelos de interpretación desarrollados, siguen siendo insuficientes y con bajo poder explicativo respecto a cuales son las variables que tienen influencia sobre la innovación. Los estudios sobre la innovación en el sector agropecuario, en gran parte se han centrado en el análisis de las tasas de adopción, lo que ha limitado las explicaciones que posibilitan entender la dinámica tecnológica seguida por los actores en su contexto específico.

Sobre esta discusión Kaine et al., (2009) consideran que lo relevante sería construir un modelo que determine los componentes del encadenamiento de gestión de la innovación en el ámbito agrícola. Vargas et al., (2009) encontraron para el caso de la agricultura protegida, los principales factores o atributos que afectan el desempeño de la innovación son: edades, escolaridad, experiencia en la actividad, confianza, 
escala y superficie de producción. Por otra parte Rojas (2000) encontró que las variables características socio económicas, psicológicas y de comportamiento social del productor son relevantes para explicar el fenómeno de la adopción de innovaciones.

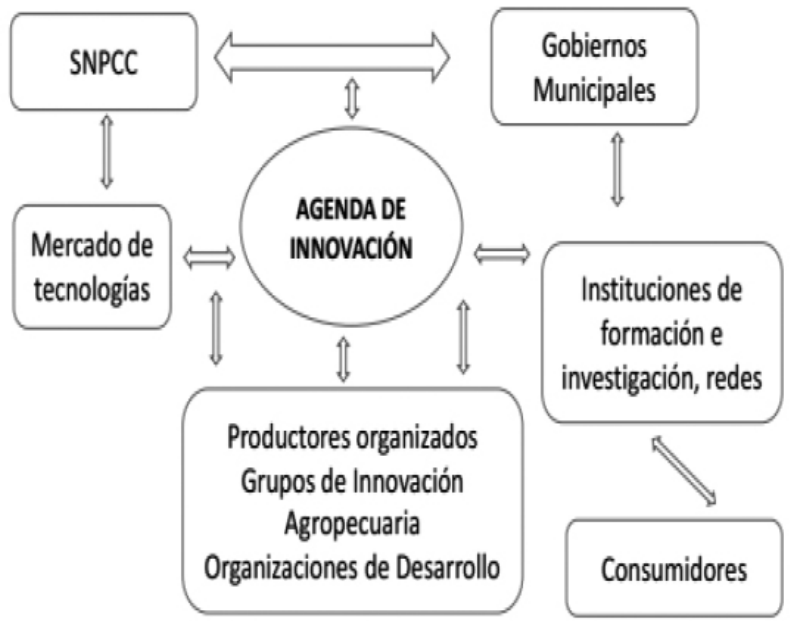

Figura 4. Actores claves en el nucleo de investigación e innovación agropecuaria

El Núcleo de investigación e innovación (NIT) como sistema territorial. Los sistemas territoriales de innovación agropecuaria deben responder a las particularidades de cada territorio, a las formas de organización de los productores, del sector público, los gobiernos municipales, las instituciones de formación e investigación y todas las expresiones de capital social que pueden incidir en el proceso de transformación de la agricultura.

A continuación se describe cada uno de los componentes que podrían participar en el NIT.

Sistema Nacional de Producción Consumo y Comercio. Es el mecanismo organizacional del Sector Público Agropecuario responsable de la implementación de las políticas, planes, programas, proyectos y acciones orientadas al desarrollo sectorial. Está integrado por el Ministerio de Agricultura (MAG), Ministerio de Economía Familiar Comunitario, Cooperativo y Asociativo (MEFCCA), Instituto de Protección y Sanidad Agropecuaria (IPSA), Instituto Nicaragüense de Tecnología Agropecuaria (INTA) y el Ministerio de Fomento Industria y Comercio (MIFIC). La acción pública en materia de sus programas debe ser pionera en términos de innovación y tienen el desafío de contribuir a la mejora de la productividad y la sustentabilidad de la agricultura. La visión de sistemas de innovación territorial podría contribuir sustancialmente al desempeño de las políticas públicas agropecuarias.
Productores individuales y mediante sus diversas formas de organización. La innovación en términos de sus resultados productivos es implementada por los productores. Son ellos los principales protagonistas de los procesos de innovación, porque además manejan el conocimiento tácito, generado por la experiencia en la gestión de sus sistemas productivos. La identificación de aquellos productores creativos que por iniciativa propia o con el respaldo de organizaciones hayan incorporado innovaciones en sus procesos de producción, podrían formar parte de los Grupos de Innovación Agropecuaria. Estos productores pueden haber construido sistemas productivos referentes para los demás productores del territorio.

Instituciones de formación e investigación, redes y otras expresiones. Este es un activo de gran importancia en los territorios, en términos de la generación de procesos de innovación. Sin embargo, a pesar que la mayoría de estas instituciones tienen las funciones de formación, investigación, extensión o proyección comunitaria, en la práctica han desaprovechado su anclaje territorial y han priorizado la formación desconectada de las necesidades de los productores. En su accionar prevalece aun la visión disciplinaria fragmentada, reflejo del modelo lineal de innovación.

Sin embargo, presentan como fortalezas talentos humanos formados en diferentes campos del desarrollo de la agricultura, infraestructura para la investigación, convenios y conexiones con instituciones internacionales de formación e investigación que podrían potencializar la capacidad de los sistemas de innovación territorial. Estas instituciones requieren ajustar sus líneas, agendas de investigación y acciones de extensión a las prioridades establecidas por los actores del sistema territorial. Su integración a la gestión de los NIT les hará avanzar en la pertinencia de sus funciones, redundará en la calidad de los futuros profesionales y encontrar el eslabón perdido entre procesos de formación, investigación y desarrollo.

Gobiernos municipales. Tienen la responsabilidad del desarrollo socio económico y ambiental en sus respectivas delimitaciones político administrativas. Tradicionalmente el mayor énfasis de los gobiernos locales se ha orientado al fortalecimiento de la infraestructura social, con poca participación en los procesos de innovación agropecuaria, por considerarla que es una atribución sectorial de las delegaciones territoriales del gobierno central. No obstante, la mejora de la multifuncionalidad de la agricultura mediante procesos de innovación, tiene impactos en las condiciones de vida de los habitantes no solamente del sector rural, sino también de las cabeceras urbanas. La inversión pública local puede mejor su desempeño mediante la creación y fortalecimiento de los NIT. 
Mercado de tecnologías. Las tecnologías generadas mediante la investigación científica, que hayan sido validadas en las condiciones de los productores de un territorio, así como aquellas que han sido creadas o modificadas por los productores innovadores deben ser ofertadas a nivel comercial, bajo un esquema de mercado justo. Se deben buscar los incentivos apropiados para desarrollar este mercado, el cual podría contribuir a la generación de empleos productivos. En la actualidad a pesar de algunos esfuerzos, este mercado es débil, provocando entre otras razones que el período entre la generación de una tecnología y su incorporación en los sistemas productivos sea lento.

Propuesta para medir innovación agropecuaria desde el NIT. En un inicio se consideraba para la medición de la innovación indicadores de innovación tecnológica, del producto y del proceso en la fase de fabricación. Posteriormente se incorporaron los cambios en las empresas de servicios, y por último se estableció como innovación los cambios que se pueden dar en las empresas en lo relativo a: producto y servicios, proceso, organización y comercialización (Manual Olso 1992, 1997, 2005).

Los indicadores propuestos para la medición de la innovación agropecuaria territorial, tienen como fundamentos conceptuales el enfoque de sistemas de innovación, el cual considera tres grandes elementos:

a) el aprendizaje generado por la interacción entre diversos actores, entre ellos, los productores y sus diversas formas de asociación, el Estado, las instituciones de formación e investigación con presencia en el territorio, las MIPYMES de servicios, organizaciones de promoción de la agricultura y mercado. La rutina de interacción debe generar productos tangibles como agendas de investigación e innovación, planes para su implementación, la creación de micro institucionalidad expresada en normas y acuerdos básicos que incentiven la interacción, puesta en común de talentos humanos, recursos materiales y financieros, entre otros.

b) en el ajuste que hace cada uno de los actores del sistema territorial, para alinear sus procesos de investigación e innovación a los requerimientos del sistema. Este es un aspecto clave, lo que significa que debe producirse a lo interno de cada actor del sistema un proceso de innovación organizacional, que les permita alinear sus políticas, programas, proyectos, agendas de investigación y estrategias de relación con el sector a las demandas de este, de manera particular de los productores y el mercado, establecidas en la agenda de innovación y sus planes de implementación.

c) Los resultados de la dinámica de interacción para el aprendizaje, el cual expresa las innovaciones generadas por el sistema en términos de la organización, mejora de procesos en la agricultura y los productos, a lo largo de cada una de las cadenas de los productos priorizados del territorio. Es decir, considera la mejora de la productividad primaria, el manejo pos cosecha y la mejora o diseño de nuevos productos con valor agregado. Considerando la sustentabilidad de la actividad agropecuaria en sus dimensiones sociales, ambientales y económicas. 
Cuadro 1. Criterios e indicadores de medición

\begin{tabular}{|c|c|c|c|}
\hline $\begin{array}{l}\text { Criterio 1. Articulación del Sistema } \\
\text { Territorial de Innovación Agropecuario }\end{array}$ & $\begin{array}{c}\text { Criterio 2. Talento humano } \\
\text { en las áreas agropecuarias } \\
\text { prioritarias }\end{array}$ & $\begin{array}{l}\text { Criterio 3. Infraestructura } \\
\text { científica tecnológica }\end{array}$ & $\begin{array}{l}\text { Criterio 4. Mercado de } \\
\text { tecnologías apropiadas } \\
\text { disponibles }\end{array}$ \\
\hline $\begin{array}{l}\text { - Participación de las tres estamentos del } \\
\text { triángulo científico tecnológico (pro- } \\
\text { ductores - sector privado mercado de } \\
\text { tecnologías, Estado e Instituciones de } \\
\text { formación e investigación) } \\
\text { - Sentido de identidad y compromiso con } \\
\text { el futuro del territorio } \\
\text { - Agenda de investigación definida y en } \\
\text { proceso de implementación } \\
\text { - Número de equipos de investigación e } \\
\text { innovación funcionando } \\
\text { - Número de Núcleos de Investigación e } \\
\text { Innovación Agropecuaria constituidos y } \\
\text { funcionando. } \\
\text { - Grupos de Innovación Integrados y fun- } \\
\text { cionando por NIT } \\
\text { - Acuerdo y apropiación de un marco } \\
\text { conceptual común } \\
\text { - Existencia de métodos de comunicación } \\
\text { que facilitan el diálogo de saberes } \\
\text { - Recursos financieros a disposición del } \\
\text { proceso innovador } \\
\text { - Recursos materiales a disposición del } \\
\text { proceso innovación }\end{array}$ & $\begin{array}{l}\text { - Investigadores relaciona- } \\
\text { das con el área agropecua- } \\
\text { rio activos por cada mil } \\
\text { habitantes } \\
\text { - Profesionales relacionados } \\
\text { con el área agropecuario } \\
\text { activos por cada mil habi- } \\
\text { tantes } \\
\text { - Técnicos relacionados con } \\
\text { el área agropecuario acti- } \\
\text { vos por cada mil habitantes } \\
\text { - Productores agropecuarios } \\
\text { innovadores activos por } \\
\text { cada mil } \\
\text { - Artesanos innovadores por } \\
\text { cada mil }\end{array}$ & $\begin{array}{l}\text { - Laboratorios ofertando servi- } \\
\text { cios técnico según vocación } \\
\text { productiva territorial } \\
\text { - Centros de Desarrollo y es- } \\
\text { taciones experimentales acti- } \\
\text { vas } \\
\text { - Fincas de referencia tecnoló- } \\
\text { gica por cada mil productores }\end{array}$ & $\begin{array}{l}\text { - Accesibilidad de tecno- } \\
\text { logías apropiadas a las } \\
\text { condiciones territoriales } \\
\text { - No. de micros y peque- } \\
\text { ños empresas MIPEs, } \\
\text { ofertando tecnologías } \\
\text { apropiadas } \\
\text { - Convenios y alianzas de } \\
\text { las MIPEs con centros } \\
\text { nacionales e internacio- } \\
\text { nales de investigación e } \\
\text { innovación }\end{array}$ \\
\hline $\begin{array}{l}\text { Criterio 5. Calidad en la gestión de los } \\
\text { procesos de innovación de las institucio- } \\
\text { nes de formación e investigación agro- } \\
\text { pecuaria y áreas afines }\end{array}$ & $\begin{array}{l}\text { - Criterio 6. Calidad en la } \\
\text { gestión de la innovación } \\
\text { desde el sector público }\end{array}$ & $\begin{array}{l}\text { - Criterio 7. Calidad en la } \\
\text { gestión de la innovación en } \\
\text { el sector de productores }\end{array}$ & $\begin{array}{l}\text { - Criterio 8. Resultados } \\
\text { de los procesos de inno- } \\
\text { vación agropecuario }\end{array}$ \\
\hline $\begin{array}{l}\text { - Líneas de investigación orientadas a la } \\
\text { agenda de investigación de los NIT } \\
\text { - Programas de estudio de pre grado y } \\
\text { post grado orientados y pertinentes a } \\
\text { prioridades de los NIT } \\
\text { - Trabajos de curso orientados a priorida- } \\
\text { des de los NIT } \\
\text { - Grupos inter y transdisciplinarios acom- } \\
\text { pañando procesos en los NIT } \\
\text { - Prácticas estudiantiles orientadas a prio- } \\
\text { ridades de los NIT } \\
\text { - Número de profesores integrados en los } \\
\text { procesos de innovación NIT } \\
\text { - Número de convenios nacionales de } \\
\text { cooperación científico técnica orienta- } \\
\text { dos a las prioridades NIT } \\
\text { - Número de convenios internacionales } \\
\text { de cooperación científico técnica orien- } \\
\text { tados a las prioridades NIT } \\
\text { - Recursos materiales orientados a los } \\
\text { procesos de gestión de la innovación } \\
\text { NIT } \\
\text { - Recursos financieros asignados a los } \\
\text { procesos de gestión de la innovación } \\
\text { NIT }\end{array}$ & $\begin{array}{l}\text { - Políticas, programas y pro- } \\
\text { yectos de fomento agrope- } \\
\text { cuario consideran el cono- } \\
\text { cimiento y las tecnologías } \\
\text { validadas en los NIT. } \\
\text { - Programas de apoyo a la } \\
\text { creación de micros y pe- } \\
\text { queñas de mercados tecno- } \\
\text { lógicos } \\
\text { - Programas de apoyo de ac- } \\
\text { ceso a mercados } \\
\text { - Disposición de fondos } \\
\text { competitivos para la inno- } \\
\text { vación agropecuaria } \\
\text { - Orientación de agenda a } \\
\text { las prioridades de los NIT } \\
\text { - Planes de desarrollo muni- } \\
\text { cipal incorporan la innova- } \\
\text { ción agropecuaria y desti- } \\
\text { nan recursos para }\end{array}$ & $\begin{array}{l}\text { - Productores participando en } \\
\text { organizaciones que promue- } \\
\text { ven la innovación } \\
\text { - Productores participando en } \\
\text { mercados internacionales } \\
\text { - Productores participando en } \\
\text { marcados nacionales } \\
\text { - Productores participando en } \\
\text { mercados locales }\end{array}$ & $\begin{array}{l}\text { - No. de innovaciones de } \\
\text { productos o de procesos } \\
\text { adoptados } \\
\text { - Incremento en la produc- } \\
\text { tividad agroecológica } \\
\text { - Mejora en la disponibili- } \\
\text { dad de alimentos } \\
\text { - Mejora en los ingresos } \\
\text { de las familias } \\
\text { - Mejora en la satisfacción } \\
\text { humana }\end{array}$ \\
\hline
\end{tabular}




\section{CONCLUSIONES}

La mayoría de las propuestas para medir indicadores de innovación responden a contextos sociales, económicos y productivos diferentes a los nuestros. En el ámbito internacional se han construidos diversas propuestas que muestran el interés de contar con instrumentos normalizados, estandarizados para estudiar y comparar la evolución de la innovación entre países y dentro de las regiones de éstos. La experiencia acerca de la medición de la innovación bajo el paradigma de los sistemas territoriales y sectoriales de innovación es escasa. Desde la perspectiva de la agricultura, los estudios se han centrado en conocer las tasas de adopción, lo que ha limitado construir explicaciones para comprender la dinámica de la innovación en su concepción amplia, incluyendo los factores que se asocian a esta. El conjunto de criterios e indicadores contenidos en este trabajo, para medir innovación, contextualiza las condiciones organizativas e institucionales del país y defiende la hipótesis que la velocidad de la innovación dependerá de la integración del conocimiento tácito y el empírico, así como en un nuevo modelo de gestión en el cual participan múltiples actores como el Estado, los productores, las instituciones de formación e investigación, los servicios privados orientados a la producción así como el entramado de organizaciones locales.

\section{REFERENCIAS BIBLIOGRÁFICAS}

Abadi, G; Pannel, D. 1999. A conceptual framework of adoption of an agricultural innovation. Agric. Econ. 21(2):145-154.

Brunet, I; Baltar, F. 2010. Desarrollo endógeno, calidad institucional. Una revisión de la teoría y algunos de sus límites. Publicado en la revista CLAD, $\mathrm{N}^{\circ} .48$.

Da Casta, S; Paez, D; Sanchez, F; Rodríguez, M. 2014. Factores favorables a la innovación en las organizaciones. Revista Psicología del Trabajo y de las Organizaciones. 30(2):67-74.

Hidalgo, A; Nuchea, A; Vizón, A; Torres, M. 2008. Los factores de la innovación tecnológica: clave de la competitividad empresarial. Dirección y organización. $\mathrm{N}^{\circ} 36$.

GRAS. 2012. Guía para evaluar la extensión rural. Foro Global para los Servicios de Asesoría Rural. Suiza. Editorial GRAS.

Sabato, J. 1975. El pensamiento latinoamericano en la problemática de ciencia - tecnología - desarrollo - dependencia. Primera edición, Buenos Aires. Ediciones Biblioteca Nacional, Argentina.

Souza, J. y colaboradores. 1993. La innovación de la innovación institucional. De lo universal, mecánico y neutral a lo contextual, interactivo y ético desde una perspectiva latinoamericana, Quito, Ecuador, Artes gráficas Silva.

Lundvall. 1992. "National System of Production, Innovation and Competence Building" Research Policy N 31. p. $213-231$.

Kaine, G, Lees, J; Wright, V. 2007. An aproach to predicting demand for an agricultural innovation. Australasian Agribusines. Review. 15:94-107.

Manual Olso. 2005. Guía para la recogida e interpretación de datos sobre innovación. Tercera edición. Traducción española: Grupo Tragsa. European Communities.

Quintero, L. 2009. Aportes teóricos para el estudio de un sistema de innovación. Revista Ciencias Administrativas y Sociales. 20(38):57-76.

Roling, N; Engel, P. 1991. It from a Knowledge System Perspective: concepts and issues. Documento presentado en el European Seminar on Knowledge Management and information Tecnology, Wageningen.

Rojas, J. 2000. Participación en el proceso de toma de decisiones para la conservación del suelo y agua: caso comunidad El Conejo, Municipio de Perote, Veracruz, México. Tesis para obtener el grado de Maestro en Ciencias, Colegio de Postgraduados, México.

Muñoz, M; Aguilar, J; Rendón, R; Altamirano, R. 2007. Análisis de la innovación en cadenas agroalimentarias. Universidad Autónoma de Chapingo - CIESTAAM / PIIAI. Primera edición, México.

Vargas, J; Palacios, M; Huitzilihuitl, J; Aguilar, J; Ocampo, J. 2015. Factores de innovación de la agricultura protegida en la región de Tulancingo, México. Revista Mexicana de Ciencias Agrícolas. 6(4):827-840. 